\title{
Spinal Epidural Abscess: A Review of Presentation, Management, and Medicolegal Implications
}

\author{
Zachary Tuvya Sharfman ${ }^{1,2}$, Yaroslav Gelfand ${ }^{1,3}$, Pryiam Shah $^{1}$, Ari Jacob Holtzman ${ }^{1,2}$, \\ Joseph Roy Mendelis ${ }^{1,2}$, Merritt Drew Kinon ${ }^{1,3}$, Jonathan David Krystal ${ }^{1,2}$, \\ Allan Brook ${ }^{1,3,4}$, Reza Yassari ${ }^{1,3}$, David Claude Kramer ${ }^{1,4}$ \\ ${ }^{1}$ Spine Surgery Outcome Group, Montefiore Medical Center, Albert Einstein College of Medicine, Bronx, NY, USA \\ ${ }^{2}$ Department of Orthopedic Surgery, Montefiore Medical Center, Albert Einstein College of Medicine, Bronx, NY, USA \\ ${ }^{3}$ Department of Neurosurgery, Montefiore Medical Center, Albert Einstein College of Medicine, Bronx, NY, USA \\ ${ }^{4}$ Department of Radiology, Montefiore Medical Center, Albert Einstein College of Medicine, Bronx, NY, USA
}

Spinal epidural abscess (SEA) is a rare condition associated with significant morbidity and mortality. Despite advances in diagnostic medicine, early recognition of SEAs remains elusive. The vague presentation of the disease, coupled with its numerous risk factors, the diagnostic requirement for obtaining advanced imaging, and the necessity of specialized care constitute extraordinary challenges to both diagnosis and treatment of SEA. Once diagnosed, SEAs require urgent or emergent medical and/or surgical management. As SEAs are a relatively rare pathology, high-quality data are limited and there is no consensus on their optimal management. This paper focuses on presenting the treatment modalities that have been successful in the management of SEAs and providing a critical assessment of how specific SEA characteristics may render one infection more amenable to primary surgical or medical interventions. This paper reviews the relevant history, epidemiology, clinical presentation, radiology, microbiology, and treatment of SEAs and concludes by addressing the medicolegal implications of delayed treatment of the disease.

Keywords: Spinal epidural abscess; Infections; Spine

\section{Introduction}

Spinal epidural abscess (SEA) was described by Giovanni Morgagni in 1761, and the first surgical intervention to address an SEA, although unsuccessful, was performed by Johann Barth in 1901 [1,2]. Successful operative interventions were performed for SEAs in the 1930s and 1940s as documented by Craig and his colleagues [3]. Historically, the incidence of SEAs has been reported at approximately 0.2-2.0 cases per 10,000 hospital admissions [4]. Over the past several decades, the reported incidence of SEAs has escalated between two- and six-fold [1,5-7]. Current prevalence estimates range between 2.2 and 20 cases per 10,000 hospital admissions, with the majority of estimates projecting 2-8 cases per 10,000 hospital admissions [7-13].

Before the advent of advanced imaging, SEA was a difficult diagnosis to substantiate, and before the introduction of advanced surgical techniques and antibiotic therapy, SEAs were almost uniformly fatal. The mortality rate currently reported in patients with SEAs is between 1.3\%

\footnotetext{
Received Dec 3, 2019; Revised Apr 12, 2020; Accepted Apr 17,2020

Corresponding author: Zachary Tuvya Sharfman

Spine Surgery Outcome Group, Montefiore Medical Center, Albert Einstein College of Medicine, 1250 Waters Place, Tower 1, 11th Floor, Bronx, New York 10461, USA

Tel: +1-347-577-4450, Fax: +1-347-577-4451, E-mail: zachsharfman@gmail.com
} 
and $31 \%$ [1,4,8,14-17]. In a large meta-analysis of patients with SEAs published 2 decades ago, Reihsaus et al. [3] reported an overall mortality rate of $16 \%$. More recent estimates, including a 2018 review of 1,094 patients with SEAs, reported adjusted mortality rates of $3.7 \%-5 \%[6,18]$. However, more than one-third of SEA survivors have poor neurological outcomes [19-21].

The classic clinical presentation of SEA was described as a triad of (1) pyrexia, (2) neck or back pain, and (3) neurological deficit. Even in the era of advanced imaging, it is important to maintain a high index of suspicion for SEA, as only $8 \%-15 \%$ of patients present with this triad $[4,6,22-$ 25]. The specificity of these clinical indicators is low [26], as one-third of patients present with isolated back pain [27] and only two-thirds of patients report symptoms in addition to back pain [28]. Fever may be noted in only one-fourth of patients [8].

Due to the vague presentation, patients are often examined by multiple physicians before making a diagnosis $[1,19,22]$. Prolonged delays in the diagnosis of SEA are common and occur in up to $89 \%$ of cases $[1,19,22]$. Timely diagnosis of SEA is paramount, as the neurological status on presentation is the single most important predictor of outcome [5,29]. To prevent irreversible neurological damage, early decompression within $24-36$ hours of the onset of the deficit is recommended $[16,20,30]$.

\section{Spread of Disease}

Pathogens invade the epidural space through the following three primary mechanisms: (1) hematogenous dissemination from a distal site, (2) extension from contiguous infected tissues, and (3) direct external inoculation $[1,6,19,26]$. The most common proposed mechanism of non-iatrogenic SEA is hematogenous dissemination, which has been purported to account for $50 \%$ of cases [6]. Of these cases, the most common proposed nidus of infection is the skin, and less commonly, the respiratory system, the urogenital system, and the oral cavity are also implicated [6]. Cervical SEA is more common in patients with intravenous drug use (IVDU) who inject them into the upper extremity and neck $[17,19,31]$. Lumbar involvement may be more common in those who inject the drugs into the legs or the groin $[9,17,19]$.

Infections in the spine are often spread through the vertebral venous plexus (VVP), which is commonly referred to as the Batson plexus, named after Dr. Oscar V. Batson who described the functional anatomy of the VVP in 1940 [32]. The VVP is directly connected to the venous system of the brain and acts as a reservoir with an important role in regulating intracranial pressures $[32,33]$. To this end, the VVP has no valves to facilitate bidirectional flow [33]. The VVP has numerous connections to the veins of the thoracoabdominal wall, the veins of the back, as well as the pulmonary, caval, and azygos systems [33,34]. In addition, the VVP has connections to the prostate, sacral, and pelvic plexuses [34]. Provided that the VVP has a rich system of interconnections throughout the body, and is a low flow/low pressure system, several SEAs can be attributed to hematogenous spread through the VVP system. Moreover, the VVP is a route for tumor dissemination, air, and cement embolism [32].

Contiguous extension from a nearby infection is responsible for approximately $30 \%$ of SEA cases, and the remainder of cases are attributable to direct inoculation during surgery and from instrumentation or procedural events [19,35-39]. Chan and Dasey [40] estimated that surgical procedures with spinal instrumentation account for $5.5 \%$ of SEAs. Other authors have reported estimates of $1.1 \%-15 \%$ [1,41]. Zimmerer et al. [41] reviewed 36 cases of SEA at a tertiary referral center where 20 of 36 patients (56\%) had SEAs secondary to either elective spinal injections (four patients) or surgery (16 patients). However, the incidence of SEAs following spinal or epidural anesthesia is extremely rare and has been reported in approximately $0.0002 \%$ of procedures [40].

\section{Predisposing Factors}

There are several factors that predispose to SEAs. The literature has reported extensively on the most relevant risk factors and comorbidities associated with SEAs (Table 1). IVDU is a common risk factor associated with SEAs, with up to $53 \%$ of cases having some association $[2,3,14,19,23,28,42,43]$. Other relevant risk factors include alcoholism, reported at $1.8 \%-22 \%$ $[2,3,14,19,23,28,42,43]$, and human immunodeficiency virus infection, reported at $1.6 \%-21.7 \%[2,14,19,23]$. Diabetes mellitus may be the most relevant risk factor in patients who do not engage in high-risk behaviors and has been reported in approximately $21 \%-42.9 \%$ of cases $[2,3,14,19,23,28,29,42,43]$. Other common medical conditions associated with SEAs include morbid obesity [2], malignancy $[2,3,23,43]$, long-term corticosteroid use [2], 


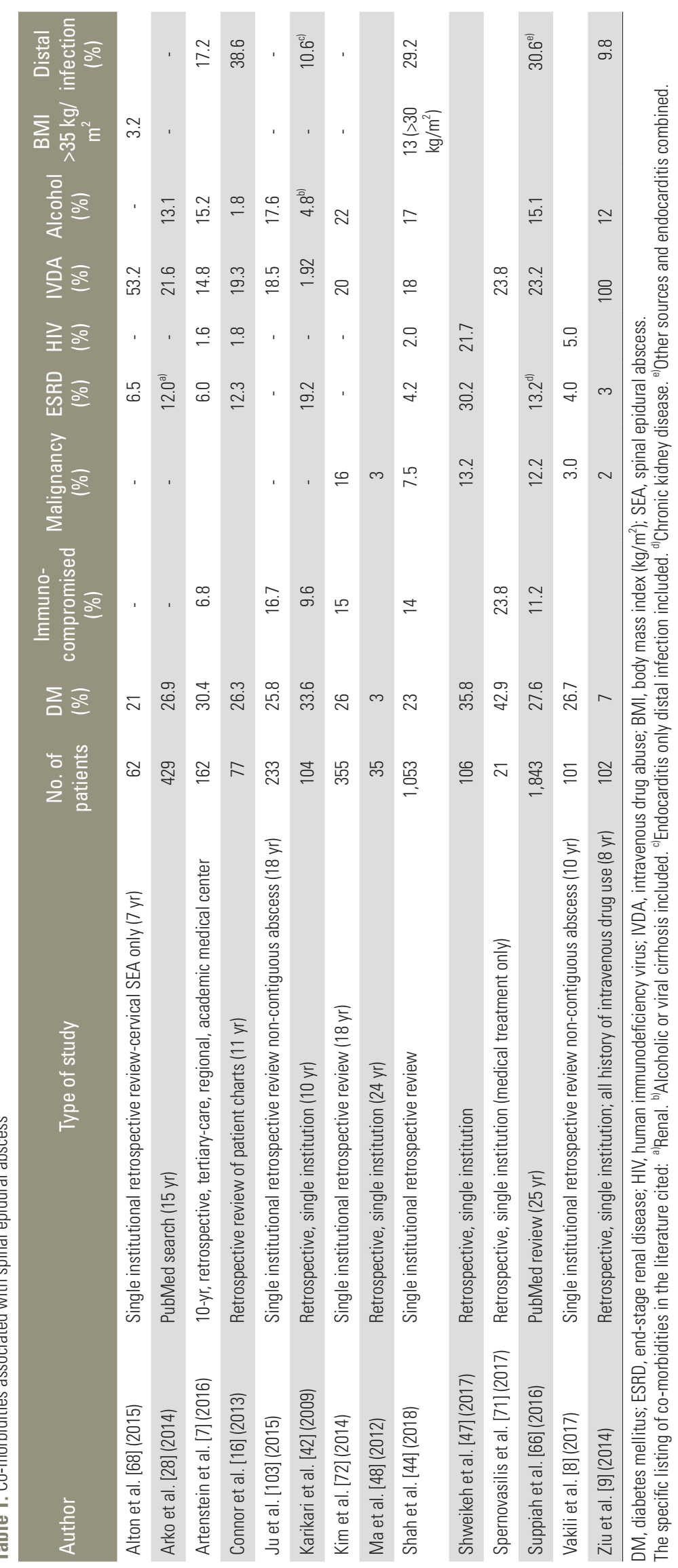


pregnancy $[3,23]$, and trauma $(10 \%-12 \%)[3,19,23,28]$. Chronic end-stage organ dysfunctions such as end-stage renal disease $[2,3,14,19,23,29,42,43]$, liver disease, and cirrhosis $[28,29,42]$ are strongly associated with SEA. Septicemia [2], distant site infection (including endocarditis) $[2,3,23,42]$, indwelling catheter [2], and invasive spinal procedures $[2,3,19]$ have also been implicated in SEA cases. Some authors report that no predisposing conditions can be identified in $20 \%-50 \%$ of cases $[2,19]$.

\section{Microbiological Specification}

The species isolated from tissue or blood culture in SEA cases is most often monobacterial (95\%); however, polymicrobial infection has also been reported in $0 \%-10.6 \%$ of patients $[8,29,44]$. Staphylococcus aureus was the most common isolate found in 50\%-93\% of cases $[3,8,28,42,45,46]$. Although some studies report low ratios of methicillin-sensitive Staphylococcus aureus (MSSA) to methicillin-resistant Staphylococcus aureus (MRSA) at $\leq 1.4[8,9,28]$, others report a higher prevalence of methicillin-resistant species $[16,31,42,46-48]$. In a retrospective review of patients with SEAs who underwent surgery, the most commonly identified microorganism was MRSA, followed by MSSA, in $43.2 \%$ and $23.7 \%$ of cases, respectively [49]. The presence of methicillin-resistant species in patients with SEAs portents a far worse prognosis than that in patients with methicillin-sensitive strains [46].

The second and third most common isolates are streptococcus species $(6 \%-15 \%)[28,44]$ and Gram-negative organisms $(4.8 \%-18.75 \%)$ [9,28,29,50]. Gram-negative organisms may be more commonly isolated from patients who use recreational intravenous drugs [51]. In an older series of 64 cases of IVDU, Pseudomonas species was isolated from $66 \%$ of patients [52]; however, this was not a common isolate in non-IVDU cohorts $(0 \%-4 \%)[9,31,42]$.

This review largely focuses on the most common bacterial culprits responsible for causing SEAs. Nevertheless, less common bacterial, fungal, and parasitic pathogens responsible for causing SEAs do exist. Any pathogen that incites a pyogenic reaction in the confined space of the spinal cord can exert a mass effect and result in neurological injury. Tuberculosis is caused by a bacterial pathogen that may result in SEAs, especially in developing countries. In cases of SEA secondary to tuberculosis, the abscess is commonly associated with tuberculous spondylitis, which is commonly referred to as Pott's disease
[53]. The mainstay of treatment includes multiagent antitubercular therapy in addition to decompression of any compressed neural elements and correction of deformity caused by the disease. Multiagent therapy typically consists of a combination of rifampin, isoniazid, ethambutol, and pyrazinamide for 2 months, followed by an additional 9-18 months of rifampin and isoniazid. Medical treatment can be further tailored to the specific sensitivities of the isolated tubercular strain.

Fungal sources have also been implicated in SEAs. In a recent review of 1,053 cases of SEA from a single institution over a period of 23 years, nine of the 1,053 patients were found to have fungal culture isolates [54]. Seven of these patients were found to have Candida species, and two had Aspergillus species [54]. Patients with fungalderived SEAs referenced in this paper were treated with antifungal agents for a minimum of 6 weeks up to 1 year unless they succumbed to their illness earlier [54]. Parasites can also be responsible for causing SEAs by inducing a local pyogenic reaction resulting in cord compression. Gopal et al. [55] reported on their case of vertebral hydatidosis causing cord compression and described the treatment, including decompression with complete cyst resection and antiparasitic agents for a minimum of 3 months. Other authors have reported about spinal hydatid and non-hydatid infections and have advocated treatment with decompression and a prolonged course of antiparasitic agents [56].

Although isolating the culprit microorganism allows for targeted antibiotic therapy, in 3.5\%-60\% of cases, no organisms are isolated from blood or tissue cultures [8$10,16,28,31,44]$. Although it is unclear whether antibiotic administration before culture affects the probability of a positive culture, some authors recommend holding antibiotics until local cultures are taken to increase the probability of a targeted treatment, unless the patient is septic [16]. Cultures from the site of infection (78.1\%-90\%) are more often successful in isolating a pathogen than blood cultures $(54.9 \%-60 \%)[16,42,57]$. It has been reported that blood cultures correlate well with tissue cultures, and targeted antibiotic therapy may be initiated based on positive blood cultures [57]. Therefore, it may be prudent to use computed tomography (CT)-guided biopsy to obtain tissue culture in patients being managed medically to optimize antibiotic therapy $[26,42]$. Table 2 lists the microbacteriology of SEAs reported in the literature. 


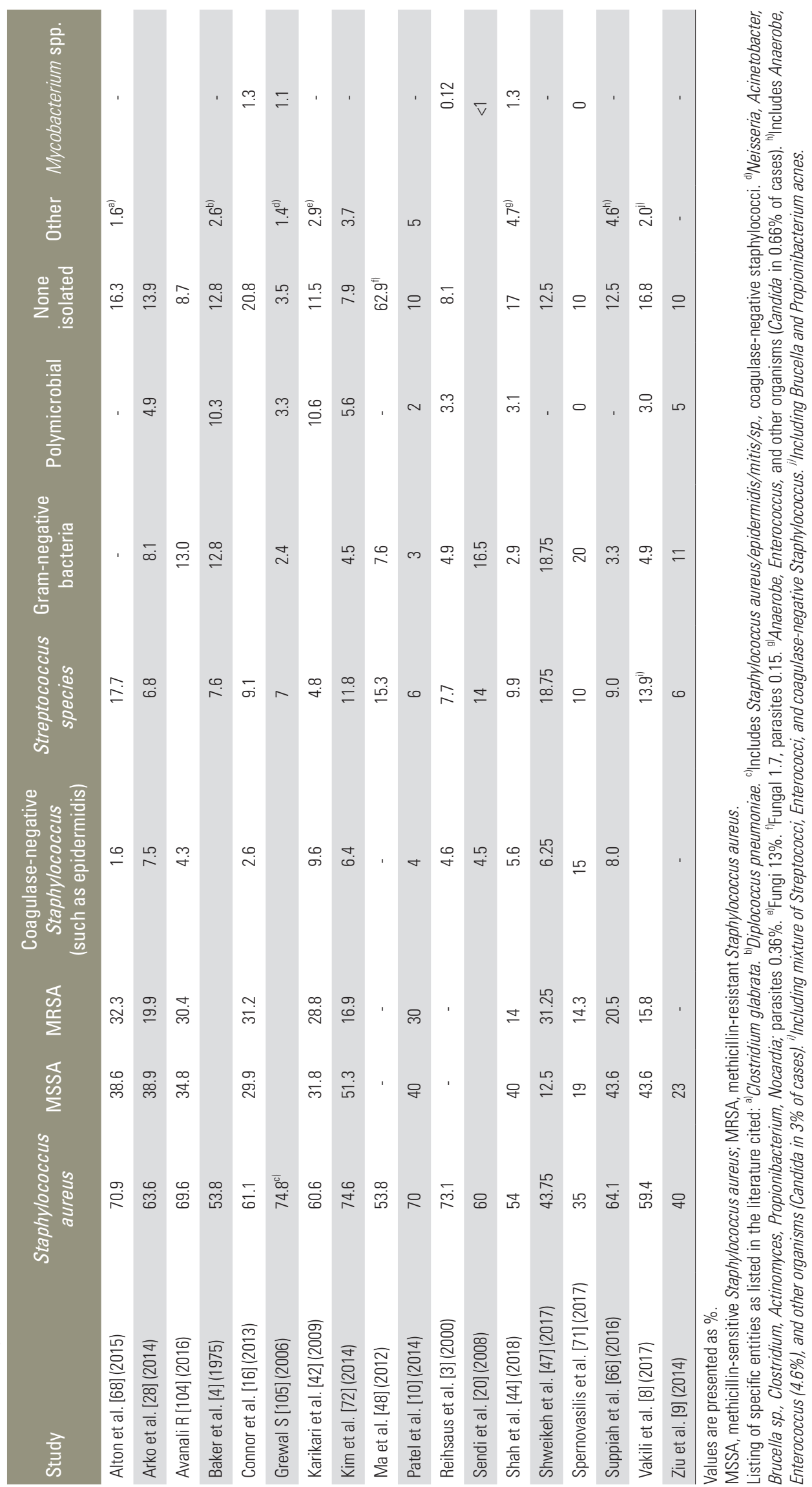




\section{Radiology}

Advanced imaging is fundamental to the diagnosis of SEA. Several algorithms designed to improve the time to diagnosis in SEAs incorporate advanced imaging at the beginning of the decision tree [19]. An emergent magnetic resonance imaging (MRI) is mandatory in patients with severe back pain accompanied by new onset or progressive neurological deficit. Other indications for an emergent or urgent MRI include severe back pain in the setting of recent bacteremia or after procedures such as spinal injection, surgery, and catheterization. Furthermore, in patients with severe back pain and elevated levels of infectious markers (erythrocyte sedimentation rate [ESR]/ C-reactive protein $[\mathrm{CRP}] /$ white blood cell $[\mathrm{WBC}]$ ), or in patients with pertinent risk factors for SEA such as diabetes, immunodeficiency, and IVDU, the threshold to obtain urgent or emergent advanced imaging should be low [19]. Advanced imaging should also be considered in patients who present repeatedly to the emergency department with back pain. It cannot be overemphasized that the diagnosis of SEA is often delayed, and the prompt initiation of MRI may remediate this problem $[22,58]$.

The ideal study to evaluate a patient with SEA is an MRI of the entire spine, with and without contrast. The characteristic findings of SEA on MRI include a high signal on T2-weighted imaging (T2WI) and a low signal on T1weighted imaging (T1WI) [59] (Fig. 1). It is important to consider that epidural abscesses are not well appreciated on precontrast T2WI, as the abscess displays similar highintensity signals as those of CSF. Precontrast T1WI is useful in detecting vertebral body and disc involvement. Postcontrast images are useful in defining the anatomical

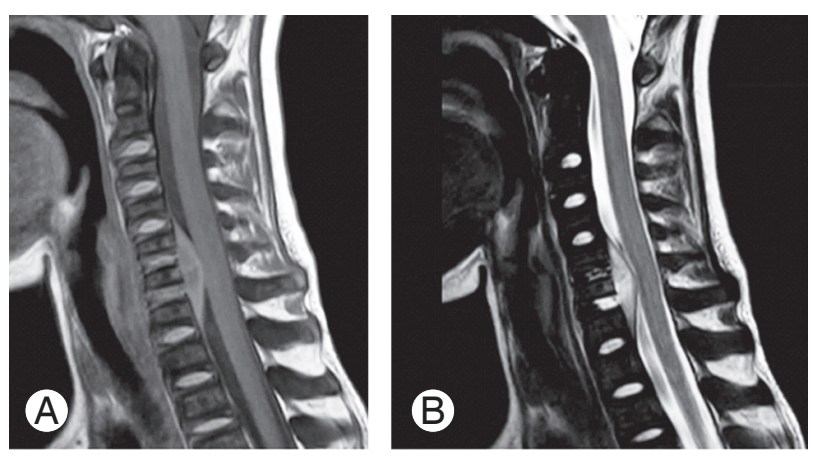

Fig. 1. (A, B) Typical MRI demonstrating low signal intensity of an anterior cervical spinal epidural abscess on T1 weighted MRI and high signal intensity on T2 weighted imaging. MRI, magnetic resonance imaging. location and the extent of an abscess, as well as the presence of spinal cord compression [59].

Broad differential diagnoses exist for SEAs, which include vertebral metastasis, epidural hematoma, disc disease (extruded or migrated discs), and vertebral osteomyelitis or osteodiscitis without SEA, among others. When the MRI, with and without intravenous contrast, is nondiagnostic, other imaging modalities may be considered.

Magnetic resonance myelography has been demonstrated to be highly sensitive in the diagnosis of SEAs $[23,60]$. CT myelography is less sensitive, although it may be the best imaging modality in cases where magnetic resonance modalities are contraindicated [20]. If possible, myelography should be avoided when there is a high index of suspicion for SEAs, due to the potential to spread the infection to the subarachnoid space [20]. In addition, CT myelography may demonstrate a myeloblock or thecal sac compression at the level of the pathology, but it may fail to demonstrate the cause of the compression, given the limitations of CT in soft tissue imaging. Nonetheless, this modality may be considered in patients with contraindications to MRI [61].

In the case that an initial imaging modality is nondiagnostic, and the index of suspicion for SEAs remains high, repeat imaging studies are mandatory. There is no consensus on the optimal timing or the modality of repeat imaging studies [20]. In cases where new-onset neurological deficits are encountered, or if a patient's symptoms do not improve after the initiation of empiric antibiotic therapy, an urgent repeat imaging should be performed. The authors of this paper recommend MRI, with and without intravenous contrast, of the entire spine as the first line of repeat imaging or CT, with and without intravenous contrast, in patients who cannot undergo MRI.

Nuclear imaging studies may also be considered in unique cases. Triple-phase bone scans with technetium99m-labeled bisphosphonates have limited sensitivity and specificity for the diagnosis of vertebral osteomyelitis; however, this can be improved when combined with a gallium-67 scan [62]. Although not currently commercially available, indium-biotin scans may have a future role in the diagnosis of SEAs or vertebral osteomyelitis, as it appears this tracer is preferentially taken up by bacteria and does not accumulate in normal bone [63]. Finally, F-fluorodeoxyglucose-positron emission tomography may be the most useful among the nuclear imaging studies for the diagnosis of SEAs, as studies have demonstrated excellent 


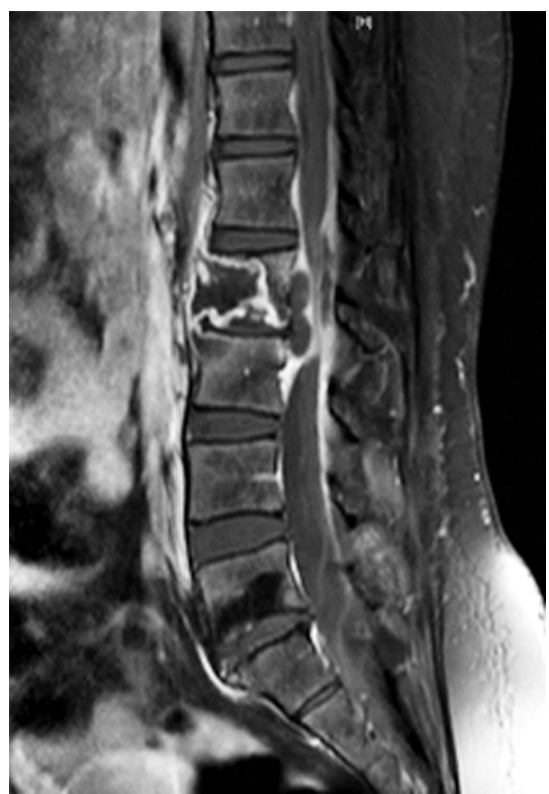

Fig. 2. Magnetic resonance imaging with contrast demonstrating a ringenhancing lumbar spinal epidural abscess.

results in differentiating between infection and degenerative changes [64,65].

Finally, the enhancement pattern observed on MRI may help direct medical or surgical management of SEAs. Homogenous enhancement may correspond to an abscess with an inflammatory tissue in the absence of a purulent collection. Peripheral or ring enhancement may indicate the presence of purulent fluid or true abscess [59]. These imaging patterns may correlate with the responsiveness of an SEA to medical versus surgical management. In case of a true abscess, the necrotic center is relatively avascular and thus has less accumulation of the contrast material, resulting in the typical ring-enhancing pattern (Fig. 2). This pathophysiology also explains why ring-enhancing abscesses are less amenable to antibiotic therapy, as the center of the abscess does not receive adequate vascular supply to treat the infection with systemic antibiotics.

\section{Medical versus Surgical Management of Spinal Epidural Abscesses}

There is a limitation of studies intended to guide the initial medical versus surgical management of SEAs, as the majority of them are small, retrospective reviews of a single institutional experience. SEA is a rare entity, and there are no studies with sufficient statistical power to detect significant differences in the outcomes of initial medical versus surgical management. Currently available treatment guidelines for SEAs are supported by case series, cohort studies, and expert opinions ranging in level of evidence from II to VI. In addition, the reported conclusions are often driven by the patient population, the spinal level of the SEA, and the location of the SEA in relation to the spinal cord.

In a case series study of 104 patients at a single center performed over a decade earlier, Karikari et al. [42] stated "Our data do not support the hypothesis that patients treated without early surgery are more likely to have a poor outcome." However, in that study, $25 \%$ of patients who were managed surgically improved, whereas only $11 \%$ of patients who were managed medically improved. Moreover, $65.4 \%$ of patients had ventral SEAs, which may be more amenable to medical management in general. Conversely, Suppiah et al. [66] performed a comprehensive review of the literature over a period of 25 years, including 1,843 patients, and identified the involvement of dorsal or circumferential SEAs in $49 \%$ of the patients. This research group favored early surgical intervention, citing it as the "optimal treatment" for patients with neurological deficits [66]. They also reported that SEAs are surgical emergencies that must be addressed immediately to avoid potential neurological damage caused by direct spinal cord compression, vascular compromise, or mechanical instability. Therefore, retrospective reviews may bias conclusions toward specific treatment modalities when the anatomical location and the orientation of the abscess vary in the reviewed population.

Concurrently, Wheeler et al. [67] performed a retrospective review of 37 SEA cases and concluded that "the current published literature does not support the widespread use of nonsurgical therapy for spinal epidural abscess." More recently, Siddiq et al. [29] concluded that "Patients with spinal epidural abscess can be safely and effectively treated with conservative medical treatment without the need for surgery." Their study may have a selection bias, as patients with more than two vertebral levels involved in the SEA were selectively treated surgically as were those with marked neurological abnormalities. Moreover, the only deaths reported in their study occurred in medically managed patients, and the authors conceded that emergency surgical decompression is the treatment of choice for patients with moderate to severe neurological symptoms at the time of presentation [29].

A retrospective study conducted by Patel et al. [10] 
reviewed the outcomes of 128 patients with SEAs treated with initial medical or surgical management. Of 51 patients initially treated medically, 21 (41\%) ultimately failed and required subsequent surgical intervention. Moreover, patients who were medically managed had higher average pretreatment motor scores; however, their return of motor function was significantly less than that in the surgically managed group [10]. The four parameters that correlated best with medical failure were (1) diabetes, (2) CRP level $>115$, (3) WBC counts $>12.5$ cells $/ \mathrm{mm}^{3}$, and (4) positive blood cultures [10].

For patients with SEAs that are exclusively confined to the cervical region, Alton et al. [68] cautioned against the sole use of medical management, as they observed a $75 \%$ failure rate in their study cohort.

Recently, there has been a change in the initial approach to SEAs. In the 2 decades preceding 1997, 87.3\% of patients with SEAs were managed surgically [28]. However, a large retrospective review of 1,095 patients with SEAs revealed that $40 \%$ of the patients were initially treated medically and $60 \%$ were initially treated surgically [28].
Caution must be applied in this approach, because medical management failed in $6 \%-49 \%$ of cases [28]. Although controversy exists regarding the best initial approach for medical versus surgical management, we have compiled a table of relevant factors affecting the success of surgical versus medical management in the setting of SEAs (Table 3). In addition to patient-specific risk factors and comorbidities, spinal cord anatomy and the location of an SEA may be vital to the success of medical or surgical management [69-71].

The majority of authors investigating SEAs view the pathology as a single infectious entity in the spine. However, one could argue that the spinal cord ends at the conus medullaris and the impact of an abscess below the cord is fundamentally different from that of an abscess above the level of the conus medullaris. One could further argue that an abscess in the lumbar spine is a fundamentally different disease process than an abscess in the cervical spine. Table 3 references the factors that may guide the initial treatment modality for patients with SEAs.

Table 3. Indication for initial medically versus surgical management in spinal epidural abscess

\begin{tabular}{|c|c|c|}
\hline Variable & Surgical & Medical \\
\hline Age & Greater than 65 years of age $[66,72,106]$; greater than 50 years of age [26] & \\
\hline Co-morbidity & Diabetes mellitus $[10,44,57,66,72,106]$; active malignancy [44] & Non-diabetic \\
\hline C-reactive protein greater than 115 & Yes [10] & C-reactive protein less than 115 \\
\hline Leukocytosis greater than 12.5 & Yes [10] & Leukocytosis less than 12 \\
\hline Microbiology & Methicillin-resistant Staphylococcus aureus $[66,71,72,106]$ & - \\
\hline Positive blood cultures & Yes [10] & No \\
\hline Sepsis & Yes [66] & No [66] \\
\hline Spinal level & $\begin{array}{l}\text { Cervical }[66,68,107] \text { or cervicothoracic [66]; more than } 5 \text { level involvement } \\
\text { [25] }\end{array}$ & $\begin{array}{l}\text { Lumbar (below conus medullaris) [66]; abscess } \\
\text { involvement is so extensive surgery is not } \\
\text { feasible [26] }\end{array}$ \\
\hline Anatomic location & Dorsal circumferential [42] & Ventral [42]; dorsal location [44] \\
\hline Spinal instability & $\begin{array}{l}\text { Instability }[28,66] \text { or pathologic or compression fracture at affected levels } \\
\text { [44] }\end{array}$ & Stable [66] \\
\hline Baseline neurologic status & $\begin{array}{l}\text { Neurologically symptomatic [16,66] especially with neurologic deficit in- } \\
\text { volving spinal cord [72] or motor weakness on presentation [44]; pelagic } \\
\text { for }<72 \mathrm{hr}[42] \text {; Sendi }[20] \text {; neurologically symptoms of less than 36-hour } \\
\text { duration [20,30,106]; a relative risk factor, but probably less predictive } \\
\text { is sensory deficit on presentation [44] }\end{array}$ & $\begin{array}{l}\text { Neurologically intact [5,43]; pelagic for }>72 \mathrm{hr} \\
{[20,42,108] \text {; complete spinal cord injury } \geq 36} \\
\text { hr }[16] \text { to } \geq 48 \text { hr prior to presentation [1] }\end{array}$ \\
\hline Decline in neurologic function & Yes [10] & No \\
\hline Radiographic findings & Ring-like enhancement on magnetic resonance imaging $[109]^{b)}$ & - \\
\hline
\end{tabular}

alBased on data of exclusively medically treated patients with low incidence of dorsal involvement. ${ }^{b}$ Enhancement of the disc, vertebral body, or para-/intraspinal soft tissues is seen in $94 \%-100 \%$ of patients with "spinal infection", however, may not be evident in severely immunocompromised patients [110]. 


\section{Abscess Location as a Guide to Treatment Approach}

The success of medical versus surgical treatment modalities is directly associated with the anatomical location, size, and the orientation of the abscess in relation to the spinal cord [42]. For instance, the management of cervical abscesses is not similar to that of lumbar abscesses and ventral abscesses, and it is also inherently different from that of dorsal and circumferential abscesses $[42,72]$. One could argue that abscesses below the spinal cord are a fundamentally different disease processes than those above the spinal cord. This is comparable to cervical disc herniation causing myelopathy (a condition that most of spine surgeons would indicate for surgical decompression) compared with lumbar disc herniation causing radiculopathy (a condition that evidence-based medicine suggests is initially managed with conservative measures). Although both these conditions are caused by disc herniations, the location of the pathology is important to the natural history of the disease and the successes of surgical versus conservative measures. Provided that the pathophysiology of neurological deficits in SEAs is believed to be largely due to compression, we postulate that the location of an abscess in the spine may dictate the success of medical versus surgical management. Table 4 outlines the anatomical distribution of SEAs reported in the literature.

Although the authors of this review ascribe to the theory that neurological deficits can largely be attributed to direct compression of the neuronal elements, other pathomechanisms for neuronal deficits have also been implicated. Local disruption of the microvasculature adjacent to an SEA secondary to venous stasis, thrombosis of spinal arteries, or local inflammatory changes may be responsible for some part of neurological deficits detected in SEAs [73-75]. This hypothesis may offer some clarification for why it is difficult to predict the timing of onset of neurological deficits in SEAs. This theory has not been collectively endorsed $[1,20]$.

Dorsal abscesses are more likely to be associated with cord compression than ventral abscesses. In a cohort study of patients who improved well with medical management, $65.4 \%$ of the SEAs were exclusively ventral and $34.6 \%$ were exclusively dorsal [42]. In that cohort, although medical management was generally recommended, patients with dorsal orientation of SEAs were much more likely to present with paraplegia or quadriplegia. Patients with lumbar involvement, who comprised the largest group in the study, did not improve with medical management [42].

Shah et al. [44] reviewed 367 patients who were initially managed medically and reported good outcomes. Their study group had significant selection bias, as the initial surgical management was an exclusion criterion. Only 8.3\% of cases involved the cervical spine, and $71 \%$ of them were below the conus medullaris [44]. Regarding the orientation of the abscess, $83 \%$ of their study patients had a ventral component, with $66 \%$ being exclusively ventral and only $16 \%$ being located exclusively in the dorsal orientation. Their study demonstrated excellent results of nonoperative management of SEAs. Physicians must note the success achieved in the study of Shah et al. [44] in medically managing ventral SEAs and SEAs below the conus medullaris with medical interventions. As the spinal cord ends at the L1-2 level, there is more space and decreased risk of compression with space-occupying lesions in the lumbar spine. Above this level, the same is not true, and medical management of SEAs may not be as successful.

In contrast to the study of Shah et al. [44] where $66 \%$ of abscesses were exclusively ventral, Alton et al. [68] performed a study of cervical SEAs in which $55 \%$ of the patients had dorsal abscess components (33.9\% dorsal and $21 \%$ circumferential). They concluded that early surgical intervention resulted in favorable outcomes compared with medical management. In addition, Patel et al. [10] advocated for initial surgical treatment of SEAs. In their study cohort, $65 \%$ of the patients had dorsal or circumferential involvement and 39.5\% had a cervical SEA.

Dorsal SEAs are much more likely to present with neurological deficits than ventral SEAs [42]. This suggests that dorsal and ventral SEAs are two distinct entities and should be treated accordingly. Neural compression occurs early in the pathogenesis of dorsal SEAs, whereas ventral SEAs are more commonly associated with discitis or osteomyelitis and present with systemic symptoms (i.e., fevers) before neurological deficits [17,21,42].

Patients with a history of IVDU present a unique set of challenges. These patients have more cervical involvement, less posterior involvement ( $29 \%$ versus $66 \%$ ), more circumferential abscesses (39\% versus $27 \%$ ), a higher rate of paralysis on presentation, a higher incidence of skip lesions (approximately 20\%), and are more likely to be on antibiotics at presentation [31]. Furthermore, they have a higher rate of early hardware failure and postoperative neurological deterioration $[31,76]$. Still, this patient 


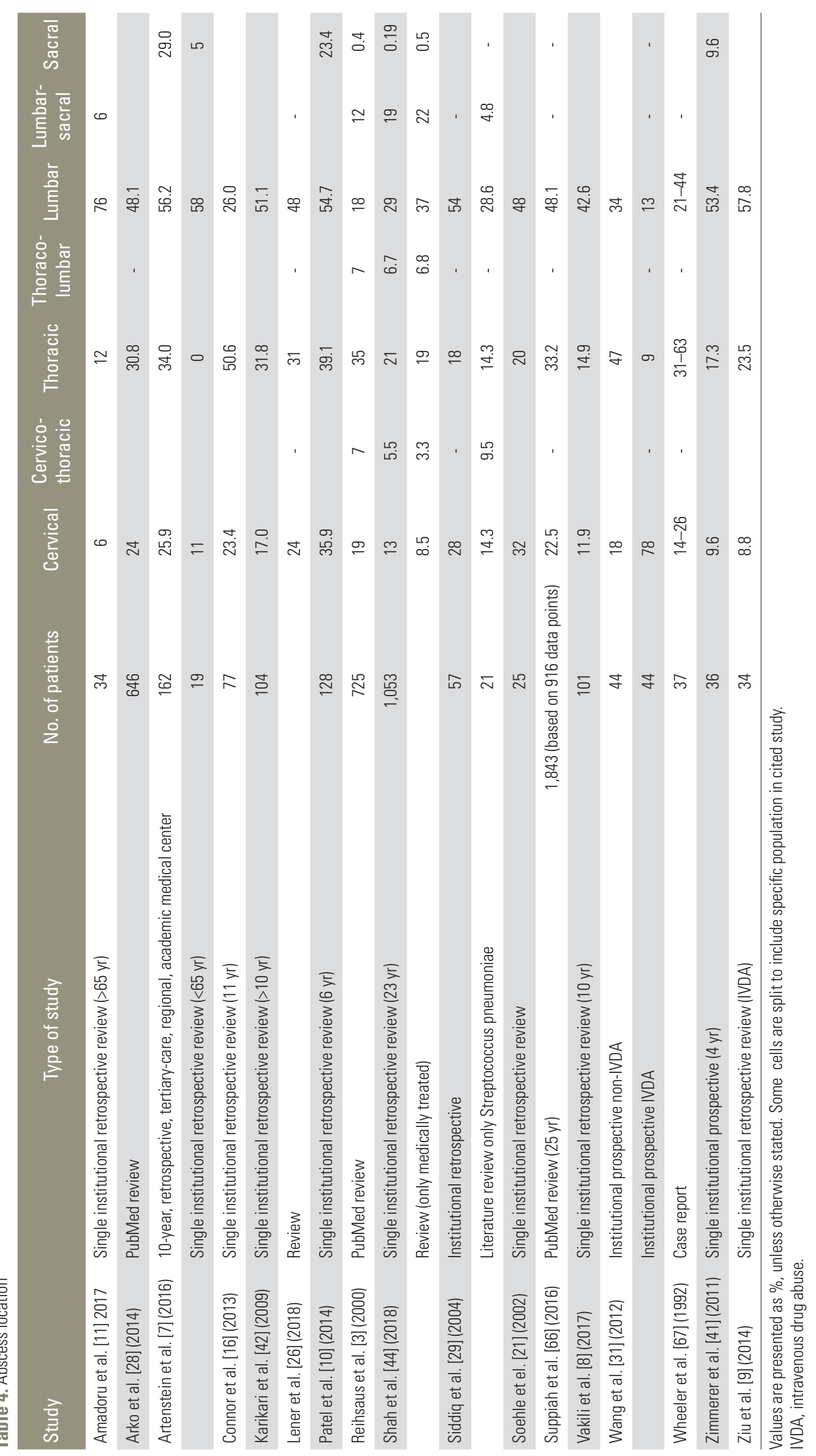


population has a higher rate of motor score improvement with surgical decompression than the non-IVDU patient population (26 versus 9 points on the American Spinal Injury Association [ASIA] impairment scale and total motor score scale) [31].

\section{Treatment Algorithm and Isolation of Organisms}

In the treatment of SEAs, it is important to recall the principles of source control and targeted antibiotic therapy. Fig. 3 depicts the authors' generalized algorithm describing our preferred approach to the diagnosis and treatment of SEAs while adhering to those principles. It is important to remember when considering this algorithm that the time to diagnosis or administer treatment and the surgeons' clinical experience weigh heavily on the optimal management of SEAs. This algorithm represents a framework for the diagnosis and management of SEAs and is not a ridged guideline.

Regardless of whether a patient will undergo interventional radiology drainage or open irrigation and debridement of an SEA, identifying the causative pathogen allows for targeted antibiotic/medical therapy. In general, culture swabs are discouraged as they often supply insufficient material for Gram staining and culture. In an ideal scenario, fluid/tissue samples should be sent for multiple aerobic, anaerobic, acid-fast bacteria, and fungal cultures. We advocate obtaining cultures in triplicate, when possible, to rule out contaminates. Although it is common practice is to grow fluid or tissue specimens on agar and broth media, recent evidence has demonstrated improved culture sensitivity when samples are grown in blood culture bottles [77]. Currently, microbiological culture remains the gold standard for identifying pathogens. However, newer techniques, including nucleic acid extraction with semiautomated sequencing, have shown promise as being more sensitive in the identification of pathogenic bacteria [78]. We recommend early multidisciplinary involvement of specialists in infectious diseases to recommend ideal techniques for the isolation of pathogens at one's specific institution.

Most of the published research recommends that antibiotics be withheld in stable patients until after biopsy is performed [79-81]. However, some literature reports no difference in culture sensitivity, even in patients who underwent biopsy at a median of 3 days after the initiation of antibiotics [81].

Guided aspiration, drainage, or biopsies may be indicated in multiple clinical situations. For instance, in frail patients who are medically unfit for surgical decompression, image-guided aspiration and decompression can provide adequate source control and accurate cultures. This minimally invasive procedure, in combination with antibiotic therapy, may be adequate to successfully treat a case of SEA. Other clinical situations where guided procedures may be appropriate include establishing a definitive diagnosis, treating a patient without neurological deficit, addressing small SEAs, and treating patients with isolated radiculopathy symptoms secondary to an SEA. Ultimately, multidisciplinary involvement, including a spinal surgeon and experts in interventional radiology, infectious diseases, and anesthesiology and the patient, will help guide the decision regarding medical versus surgical management. This topic is further explored in the section regarding medical versus surgical management of SEAs.

\section{Infectious Markers and Monitoring Outcomes}

The role of inflammatory markers is of great importance to both diagnosis and monitoring of SEAs. The finding of an elevated WBC count in a routine laboratory testing can be indicative of an underlying inflammation or infection; however, it has limited sensitivity or specificity [82]. More sensitive markers such as serum ESR and serum CRP, have been routinely used to screen for and monitor inflammatory states. Various literature has demonstrated that a serum ESR level $>30 \mathrm{~mm} / \mathrm{hr}$ and a serum CRP level $>10 \mathrm{mg} / \mathrm{L}$ achieved a sensitivity of $>95 \%$ [83]. Both are useful markers for detecting an underlying infection, with CRP being more specific among the two. Recent research has also suggested that serum D-dimer may be even more sensitive than CRP in diagnosing infection, although less specific [84].

CRP may also be a useful marker for monitoring the resolution or nonresolution of infection after the initiation of treatment. With the presence of an inflammatory stimulus, the level of CRP is expected to double every 8 hours and peak at 36-50 hours. After the removal of that stimulus, the CRP level falls rapidly with a half-life of 19 hours [85]. We recommend monitoring the CRP level every $24-48$ hours after the initiation of treatment, as values can be expected to decline rapidly after the initiation of appropriate treatment.

Procalcitonin has shown promise in distinguishing bac- 


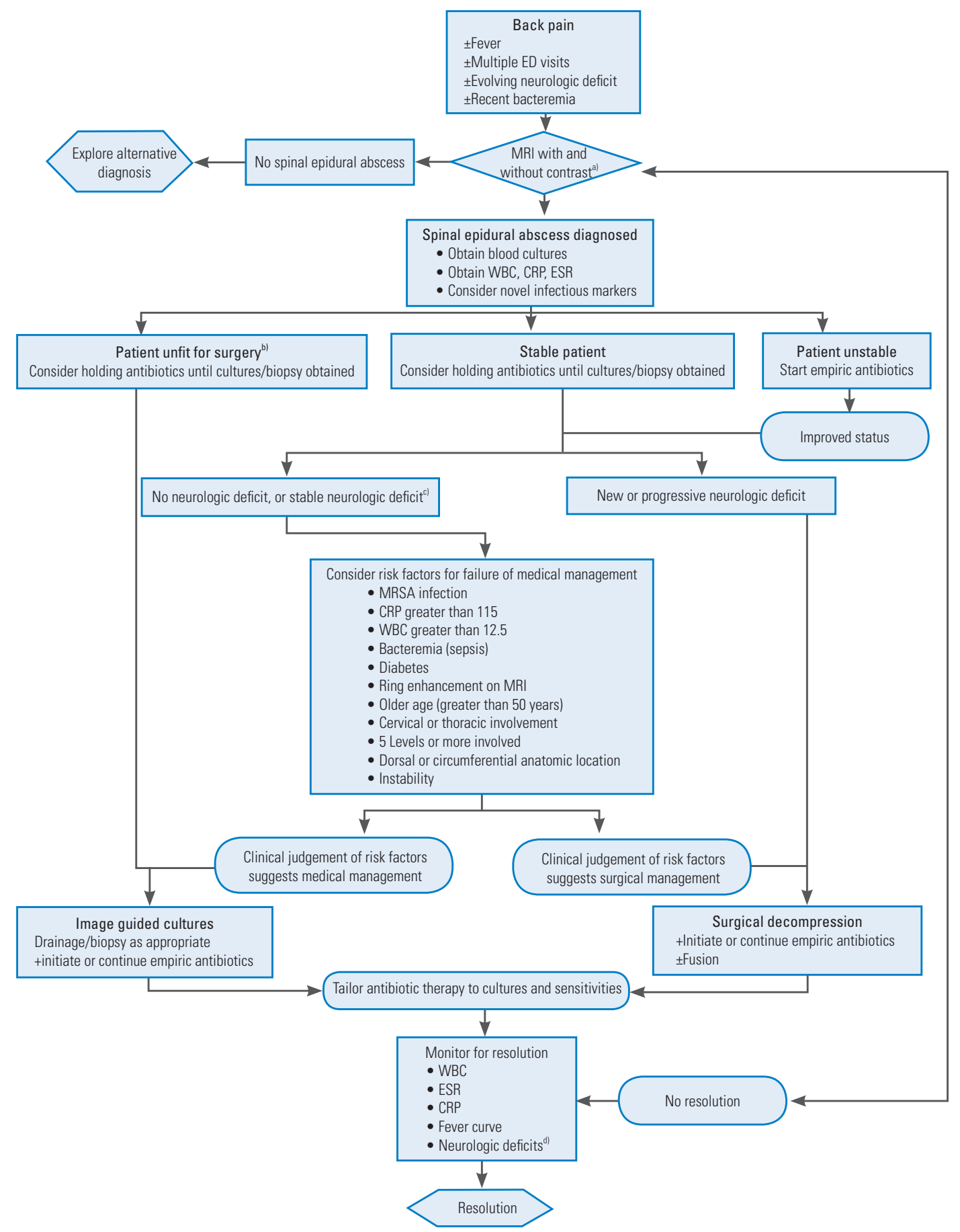

Fig. 3. Generalized algorithm describing our preferred approach to the diagnosis and treatment of spinal epidural abscess. It is important to remember when considering this algorithm that time to diagnosis/treatment and the surgeons clinical experience weigh heavily on the optimal management of spinal epidural abscess. This algorithm represents a framework for the diagnosis and management of spinal epidural abscess not a ridged guideline. ED, emergency department; MRI, magnetic resonance imaging; WBC, white blood cell; CRP, C-reactive protein; ESR, erythrocyte sedimentation rate. ${ }^{\text {al } M R I}$ with and without intravenous contrast of the entire

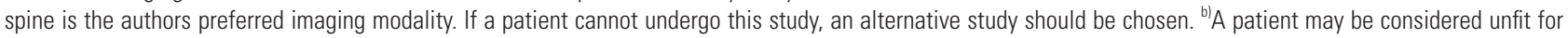
surgery if he/she (1) refuses any operative intervention, (2) has considerable medical risk factors, which are determined with input from the anesthesiologists that the risks of surgical intervention outweigh the potential benefits, and (3) has a concurrent medical condition which requires urgent intervention that surpasses the spinal

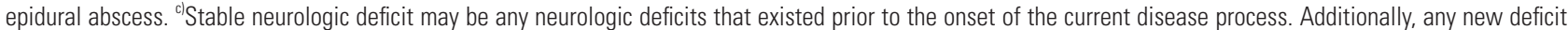
which is stable may not necessitate operative intervention. Some surgeons may choose to treat neurologic deficits consistent with radiculopathy with initial medical management. Again, considerations of the timing of presentation, and the severity of disease are not covered in this algorithm and weigh heavily on the decision to procced. Finally, there is no substitute for a surgeon's clinical experience in the management of spinal epidural abscess. ${ }^{d / N e w}$ onset neurologic deficits after management is initiated or the worsening of deficits are important clinical signs that should be investigated thoroughly possibly with repeat imaging. If neurologic deficits do not resolve after treatment that is not necessarily a sign of failed management. 
terial and nonbacterial infections; however, it has lower sensitivity than CRP in diagnosing SEAs [86]. Other novel markers such as high-sensitivity-CRP, presepsin, and alpha-defensin have not been thoroughly investigated at this point, and specific diagnostic criteria cannot be recommended. Nevertheless, these markers may be of use in diagnosing or monitoring SEAs.

When possible, antibiotic therapy should be tailored to the offending pathogen and delivered via the route of highest penetrance, which is most often intravenous. There is some evidence indicating that oral antibiotics are as effective as intravenous antibiotics in the management of infection; however, the use of oral antibiotics in the initial or empiric treatment of SEAs is not common [87]. When no pathogen is identified, empiric antibiotics, including vancomycin plus ceftriaxone or Zosyn, are often the first line of empiric antibiotic treatment. When there is no response to these medications, the empiric treatment should be altered with co-management by specialists in infectious diseases.

Although the duration of treatment is variable, it should typically not be shorter than 6 weeks. Treatment duration can vary significantly between 6 weeks and multiple months depending on the type of infection. Moreover, in some cases, indefinite suppressive antibiotic therapy may be necessary. However, most often, CRP levels can be trended throughout the medical treatment. As CRP levels normalize, any underlying osteomyelitis or discitis is typically treated with a prolonged course of antibiotics. When an appropriate decrease in CRP levels is not observed, and the patient has new clinical signs or symptoms of disease progression or other infectious markers become elevated, further workup and management may be necessary. Fig. 3 outlines the typical treatment algorithm for patients with SEAs, including monitoring for the resolution of disease.

\section{Instrumentation during Infection}

An isolated SEA is not generally associated with deformity or instability. However, when instrumented decompression is indicated, infection should not prohibit hardware placement. Decompression, with or without instrumentation, has been reported to have equivalent rates of reoperation and resolution of the infection $[49,88,89]$, even at long-term follow-up [90,91].

In the setting of osteomyelitis, Chen et al. [92] have stratified the need for instrumentation to include (1) insta- bility, (2) significant destruction of endplates, (3) abscess formation, (4) new neurological deficit, (5) local kyphosis, (6) septic pseudoarthrosis, and (7) refractory pain or infection. The literature strongly supports the paradigm that if neurological instability exists, decompression and instrumented spinal stabilization may be performed in a single stage, even in the presence of active infection [89-91,93-95]. If instrumentation is used, long-term clearance of infection may be more likely with titanium or titanium alloy implants than with stainless steel implants [93]. It appears that instrumentation of the infected spine may be a safe practice and should be considered when the spinal stability is compromised, even in cases of resistant organisms such as MRSA [49]. Immunosuppressed patients with vertebral osteomyelitis have a low risk of $3.13 \%$ (1/32) of developing recurrence after decompression and instrumentation [96]. Reoperation rates are similar between the decompression alone (19.44\%) and instrumented decompression (17.1\%) groups in the setting of spinal infection [49]. Other authors have reported that in the setting of SEAs, instrumented decompression not only has equivalent success rates as those of uninstrumented decompression, but it also may be associated with decreased mortality [94].

\section{Neurological Outcomes}

Even when SEAs are well managed, neurological outcomes can be poor. In general, an early operative intervention is recommended, as delays in surgical intervention are associated with increased morbidity and mortality [30]. In a retrospective review of operative versus nonoperative management of SEAs, Connor et al. [16] demonstrated that approximately $80 \%$ of patients $(79.2 \%)$ had improvements in neurological deficits following decompression. The authors also demonstrated that advanced patient age and more severe preoperative deficits correlated with poor neurological outcomes. Their study is only one example of the numerous studies conducted by authors who advocate for early surgical intervention to improve neurological and survival outcomes $[16,68,97,98]$. Perhaps most notably, Patel et al. [10] demonstrated that early surgery improved the neurological outcomes compared with surgical management delayed by a trial of medical management. In addition, more than $41 \%$ of patients in that cohort ultimately failed medical management and required surgical decompression. The patient group that ultimately failed medical management had net deterioration in their ASIA 
motor score of 14.86 points compared to that in the other groups who demonstrated improvements [10]. In addition to the potentially devastating neurological outcomes associated with SEAs, there is potential for structural spinal sequelae, complications associated with the dissemination of infectious materials, such as endocarditis, or even death. The reported mortality rates associated with SEAs range from $3 \%$ to $25 \%$ [99].

\section{Medicolegal Cost}

Throughout this article, we have emphasized the importance of early diagnosis and treatment of SEAs before the onset of permanent neurological damage. In addition to the immeasurable cost of human suffering associated with the sequelae of delayed treatment in SEAs, there is a substantial medicolegal exposure [100].

It has been observed that $61 \%$ of law suits pertaining to SEAs are settled outside of court or end with a ruling that favors the plaintiff [100]. The mean cost of a ruling in favor of a plaintiff is US $\$ 5,277,468 \pm$ US $\$ 6,348,462$, and settlements average at US $\$ 1,914,265 \pm$ US $\$ 1,313,322$ [100]. Plaintiffs who suffer a significant neurological morbidity are more likely to be awarded substantial settlements averaging US $\$ 4,699,772 \pm$ US $\$ 5,369,684$ [100]. Delayed time to imaging (33.75 versus 4 days), expert consultation (32.7 versus 2.8 days), and surgical intervention (33.5 versus 18.9 days) are the key factors associated with medical liability [101]. Specifically, delayed diagnosis, delayed treatment [102], and paralysis are independent risk factors for legal decisions in favor of the plaintiff [100]. Plaintiff verdicts were more common in patients who sustained significant neurological deficits secondary to their disease, and these plaintiffs were also awarded larger rulings [102].

When reviewing medicolegal liability, it is important to understand the factors that correlate with "failure to recover." In the treatment of SEAs, "failure to recover" is affected by age (the single most important predictive factor), paralysis, renal failure, and a patient's insurance status [15]. Medicare and underinsured patients fare far worse than those with private insurance [15]. Faber et al. [13] classified patients with SEAs into early surgery, delayed surgery, and medical management treatment groups. Patients who received early surgical interventions within 48 hours of admission were more likely to be privately insured [13]. This type of selection bias, either conscious or unconscious, has an enormous impact on patient morbidity and mortality and adds to a significant medicolegal exposure for physicians.

\section{Conclusions}

Early diagnosis and emergent management are vital in the successful treatment of SEAs. Educating physicians and instituting policies and practices to obtain urgent advanced imaging in patients with back pain or newonset neurological symptoms or in patients who return to the emergency department for continued back pain, even without neurological symptoms, are vital to the early diagnosis of SEAs. Once diagnosed, assessing an abscess' characteristics and classifying it based on its location, size, appearance on imaging, aberrant laboratory values, the patient's comorbidity profile, and the association with neurological symptoms may determine whether an SEA is amenable to initial medical or surgical management. Early subspecialist involvement, including consultations in infectious disease, orthopedic or neurosurgery, and rehabilitation medicine, is vital. Continued research and high-quality studies would further illuminate the optimal management of SEAs.

\section{Conflict of Interest}

No potential conflict of interest relevant to this article was reported.

\section{References}

1. Tuchman A, Pham M, Hsieh PC. The indications and timing for operative management of spinal epidural abscess: literature review and treatment algorithm. Neurosurg Focus 2014;37:E8.

2. Tompkins M, Panuncialman I, Lucas P, Palumbo M. Spinal epidural abscess. J Emerg Med 2010;39:38490.

3. Reihsaus E, Waldbaur H, Seeling W. Spinal epidural abscess: a meta-analysis of 915 patients. Neurosurg Rev 2000;23:175-204.

4. Baker AS, Ojemann RG, Swartz MN, Richardson EP Jr. Spinal epidural abscess. N Engl J Med 1975;293:463-8.

5. Strauss I, Carmi-Oren N, Hassner A, Shapiro M, Giladi M, Lidar Z. Spinal epidural abscess: in search of reasons for an increased incidence. Isr Med Assoc J 
2013;15:493-6.

6. Darouiche RO. Spinal epidural abscess. N Engl J Med 2006;355:2012-20.

7. Artenstein AW, Friderici J, Holers A, Lewis D, Fitzgerald J, Visintainer P. Spinal epidural abscess in adults: a 10-year clinical experience at a tertiary care academic medical center. Open Forum Infect Dis 2016;3:ofw191.

8. Vakili M, Crum-Cianflone NF. Spinal epidural abscess: a series of 101 cases. Am J Med 2017;130:1458-63.

9. Ziu M, Dengler B, Cordell D, Bartanusz V. Diagnosis and management of primary pyogenic spinal infections in intravenous recreational drug users. Neurosurg Focus 2014;37:E3.

10. Patel AR, Alton TB, Bransford RJ, Lee MJ, Bellabarba CB, Chapman JR. Spinal epidural abscesses: risk factors, medical versus surgical management, a retrospective review of 128 cases. Spine J 2014;14:326-30.

11. Amadoru S, Lim K, Tacey M, Aboltins C. Spinal infections in older people: an analysis of demographics, presenting features, microbiology and outcomes. Intern Med J 2017;47:182-8.

12. Ho EL. Infectious etiologies of myelopathy. Semin Neurol 2012;32:154-60.

13. Farber SH, Murphy KR, Suryadevara CM, et al. Comparing outcomes of early, late, and non-surgical management of intraspinal abscess. J Clin Neurosci 2017;36:64-71.

14. DeSanto J, Ross JS. Spine infection/inflammation. Radiol Clin North Am 2011;49:105-27.

15. Schoenfeld AJ, Wahlquist TC. Mortality, complication risk, and total charges after the treatment of epidural abscess. Spine J 2015;15:249-55.

16. Connor DE Jr, Chittiboina P, Caldito G, Nanda A. Comparison of operative and nonoperative management of spinal epidural abscess: a retrospective review of clinical and laboratory predictors of neurological outcome. J Neurosurg Spine 2013;19:119-27.

17. Pradilla G, Nagahama Y, Spivak AM, Bydon A, Rigamonti D. Spinal epidural abscess: current diagnosis and management. Curr Infect Dis Rep 2010;12:484-91.

18. Du JY, Schell AJ, Kim CY, Trivedi NN, Ahn UM, Ahn NU. 30-Day mortality following surgery for spinal epidural abscess: incidence, risk factors, predictive algorithm, and associated complications. Spine (Phila Pa 1976) 2019;44:E500-9.
19. Bond A, Manian FA. Spinal epidural abscess: a review with special emphasis on earlier diagnosis. Biomed Res Int 2016;2016:1614328.

20. Sendi P, Bregenzer T, Zimmerli W. Spinal epidural abscess in clinical practice. QJM 2008;101:1-12.

21. Soehle M, Wallenfang T. Spinal epidural abscesses: clinical manifestations, prognostic factors, and outcomes. Neurosurgery 2002;51:79-85.

22. Davis DP, Wold RM, Patel RJ, et al. The clinical presentation and impact of diagnostic delays on emergency department patients with spinal epidural abscess. J Emerg Med 2004;26:285-91.

23. Hlavin ML, Kaminski HJ, Ross JS, Ganz E. Spinal epidural abscess: a ten-year perspective. Neurosurgery 1990;27:177-84.

24. Moatz B, Michael K, Rhee JM. Spinal epidural abscesses: diagnosis and current treatment options. Semin Spine Surg 2016;28:143-9.

25. Tahir MZ, Hassan RU, Enam SA. Management of an extensive spinal epidural abscess from C-1 to the sacrum. Case report. J Neurosurg Spine 2010;13:780-3.

26. Lener S, Hartmann S, Barbagallo GM, Certo F, Thome C, Tschugg A. Management of spinal infection: a review of the literature. Acta Neurochir (Wien) 2018;160:487-96.

27. Curry WT Jr, Hoh BL, Amin-Hanjani S, Eskandar EN. Spinal epidural abscess: clinical presentation, management, and outcome. Surg Neurol 2005;63:364-71.

28. Arko L 4th, Quach E, Nguyen V, Chang D, Sukul V, Kim BS. Medical and surgical management of spinal epidural abscess: a systematic review. Neurosurg Focus 2014;37:E4.

29. Siddiq F, Chowfin A, Tight R, Sahmoun AE, Smego RA Jr. Medical vs surgical management of spinal epidural abscess. Arch Intern Med 2004;164:2409-12.

30. Shweikeh F, Saeed K, Bukavina L, Zyck S, Drazin D, Steinmetz MP. An institutional series and contemporary review of bacterial spinal epidural abscess: current status and future directions. Neurosurg Focus 2014;37:E9.

31. Wang Z, Lenehan B, Itshayek E, et al. Primary pyogenic infection of the spine in intravenous drug users: a prospective observational study. Spine (Phila Pa 1976) 2012;37:685-92.

32. Nathoo N, Caris EC, Wiener JA, Mendel E. History of the vertebral venous plexus and the significant 
contributions of Breschet and Batson. Neurosurgery 2011;69:1007-14.

33. Groen RJ, Groenewegen HJ, van Alphen HA, Hoogland PV. Morphology of the human internal vertebral venous plexus: a cadaver study after intravenous Araldite CY 221 injection. Anat Rec 1997;249:285-94.

34. Groen RJ, du Toit DF, Phillips FM, et al. Anatomical and pathological considerations in percutaneous vertebroplasty and kyphoplasty: a reappraisal of the vertebral venous system. Spine (Phila Pa 1976) 2004;29:1465-71.

35. Chan JJ, Oh JJ. A rare case of multiple spinal epidural abscesses and cauda equina syndrome presenting to the emergency department following acupuncture. Int J Emerg Med 2016;9:22.

36. Godhania V. Lumbar spine osteomyelitis and epidural abscess formation secondary to acupuncture. J Surg Case Rep 2016;2016:rjw035.

37. Lee JH, Cho JH, Jo DJ. Cervical epidural abscess after cupping and acupuncture. Complement Ther Med 2012;20:228-31.

38. Reynolds F. Neurological infections after neuraxial anesthesia. Anesthesiol Clin 2008;26:23-52.

39. Okano K, Kondo H, Tsuchiya R, Naruke T, Sato M, Yokoyama R. Spinal epidural abscess associated with epidural catheterization: report of a case and a review of the literature. Jpn J Clin Oncol 1999;29:49-52.

40. Chan YC, Dasey N. Iatrogenic spinal epidural abscess. Acta Chir Belg 2007;107:109-18.

41. Zimmerer SM, Conen A, Muller AA, et al. Spinal epidural abscess: aetiology, predisponent factors and clinical outcomes in a 4-year prospective study. Eur Spine J 2011;20:2228-34.

42. Karikari IO, Powers CJ, Reynolds RM, Mehta AI, Isaacs RE. Management of a spontaneous spinal epidural abscess: a single-center 10-year experience. Neurosurgery 2009;65:919-23.

43. Ropper AE, Ropper AH. Acute spinal cord compression. N Engl J Med 2017;376:1358-69.

44. Shah AA, Ogink PT, Harris MB, Schwab JH. Development of predictive algorithms for pre-treatment motor deficit and 90-day mortality in spinal epidural abscess. J Bone Joint Surg Am 2018;100:1030-8.

45. Russell NA, Vaughan R, Morley TP. Spinal epidural infection. Can J Neurol Sci 1979;6:325-8.

46. Huang PY, Chen SF, Chang WN, et al. Spinal epidur- al abscess in adults caused by Staphylococcus aureus: clinical characteristics and prognostic factors. Clin Neurol Neurosurg 2012;114:572-6.

47. Shweikeh F, Hussain M, Sangtani A, et al. Cervical spine epidural abscess: a single center analytical comparison to the literature. Spinal Cord Ser Cases 2017;3:17036.

48. Ma H, Kim I. Clinical outcomes of spinal epidural abscess. Korean J Spine 2012;9:6-11.

49. Bydon M, De la Garza-Ramos R, Macki M, et al. Spinal instrumentation in patients with primary spinal infections does not lead to greater recurrent infection rates: an analysis of 118 cases. World Neurosurg 2014;82:e807-14.

50. Falade OO, Antonarakis ES, Kaul DR, Saint S, Murphy PA. Clinical problem-solving: beware of first impressions. N Engl J Med 2008;359:628-34.

51. Kaufman DM, Kaplan JG, Litman N. Infectious agents in spinal epidural abscesses. Neurology 1980;30:844-50.

52. Sapico FL, Montgomerie JZ. Vertebral osteomyelitis in intravenous drug abusers: report of three cases and review of the literature. Rev Infect Dis 1980;2:196-206.

53. Jia-Jia S, Zhi-Yong S, Zhong-Lai Q, Hui-Lin Y, Xiao$\mathrm{Yu} \mathrm{Z}$. Tuberculous spondylitis after vertebral augmentation: a case report with a literature review. J Int Med Res 2018;46:916-24.

54. Yang H, Shah AA, Nelson SB, Schwab JH. Fungal spinal epidural abscess: a case series of nine patients. Spine J 2019;19:516-22.

55. Gopal N, Chauhan S, Yogesh N. Primary spinal extradural hydatid cyst causing spinal cord compression. Indian J Orthop 2007;41:76-8.

56. Xia Y, Ju Y, Liu JP, Chen LY. Common spinal parasites. Turk Neurosurg 2019;29:409-13.

57. Krishnamohan P, Berger JR. Spinal epidural abscess. Curr Infect Dis Rep 2014;16:436.

58. Alerhand S, Wood S, Long B, Koyfman A. The timesensitive challenge of diagnosing spinal epidural abscess in the emergency department. Intern Emerg Med 2017;12:1179-83.

59. Thurnher MM. Spinal infections. In: Van Goethem J, van den Hauwe L, Parizel PM, editors. Spinal imaging: diagnostic imaging of the spine and spinal cord. Berlin: Springer Berlin Heidelberg; 2007. p. 521-41.

60. Rigamonti D, Liem L, Sampath P, et al. Spinal epidural abscess: contemporary trends in etiology, evalua- 
tion, and management. Surg Neurol 1999;52:189-96.

61. Gerstein N. Spinal epidural abscess. N Engl J Med 2007;356:638.

62. Palestro CJ. Radionuclide imaging of osteomyelitis. Semin Nucl Med 2015;45:32-46.

63. Lazzeri E, Erba P, Perri M, et al. Scintigraphic imaging of vertebral osteomyelitis with 111 in-biotin. Spine (Phila Pa 1976) 2008;33:E198-204.

64. Stumpe KD, Zanetti M, Weishaupt D, Hodler J, Boos N, von Schulthess GK. FDG positron emission tomography for differentiation of degenerative and infectious endplate abnormalities in the lumbar spine detected on MR imaging. AJR Am J Roentgenol 2002;179:1151-7.

65. Fuster D, Sola O, Soriano A, et al. A prospective study comparing whole-body FDG PET/CT to combined planar bone scan with 67Ga SPECT/CT in the diagnosis of spondylodiskitis. Clin Nucl Med 2012;37:827-32.

66. Suppiah S, Meng Y, Fehlings MG, Massicotte EM, Yee A, Shamji MF. How best to manage the spinal epidural abscess?: a current systematic review. World Neurosurg 2016;93:20-8.

67. Wheeler D, Keiser P, Rigamonti D, Keay S. Medical management of spinal epidural abscesses: case report and review. Clin Infect Dis 1992;15:22-7.

68. Alton TB, Patel AR, Bransford RJ, Bellabarba C, Lee MJ, Chapman JR. Is there a difference in neurologic outcome in medical versus early operative management of cervical epidural abscesses? Spine J 2015; 15:10-7.

69. Adogwa O, Karikari IO, Carr KR, et al. Spontaneous spinal epidural abscess in patients 50 years of age and older: a 15-year institutional perspective and review of the literature: clinical article. J Neurosurg Spine 2014;20:344-9.

70. Lu CH, Chang WN, Lui CC, Lee PY, Chang HW. Adult spinal epidural abscess: clinical features and prognostic factors. Clin Neurol Neurosurg 2002;104:306-10.

71. Spernovasilis N, Demetriou S, Bachlitzanaki M, et al. Characteristics and predictors of outcome of spontaneous spinal epidural abscesses treated conservatively: a retrospective cohort study in a referral center. Clin Neurol Neurosurg 2017;156:11-7.

72. Kim SD, Melikian R, Ju KL, et al. Independent predictors of failure of nonoperative management of spinal epidural abscesses. Spine J 2014;14:1673-9.

73. Pradilla G, Ardila GP, Hsu W, Rigamonti D. Epidural abscesses of the CNS. Lancet Neurol 2009;8:292-300.

74. Shah NH, Roos KL. Spinal epidural abscess and paralytic mechanisms. Curr Opin Neurol 2013;26:314-7.

75. Darouiche RO, Hamill RJ, Greenberg SB, Weathers SW, Musher DM. Bacterial spinal epidural abscess: review of 43 cases and literature survey. Medicine (Baltimore) 1992;71:369-85.

76. DiGiorgio AM, Stein R, Morrow KD, Robichaux JM, Crutcher CL, Tender GC. The increasing frequency of intravenous drug abuse-associated spinal epidural abscesses: a case series. Neurosurg Focus 2019;46:E4.

77. Peel TN, Dylla BL, Hughes JG, et al. Improved diagnosis of prosthetic joint infection by culturing periprosthetic tissue specimens in blood culture bottles. mBio 2016;7:e01776-15.

78. Fihman V, Hannouche D, Bousson V, et al. Improved diagnosis specificity in bone and joint infections using molecular techniques. J Infect 2007;55:510-7.

79. Zimmerli W. Clinical practice: vertebral osteomyelitis. N Engl J Med 2010;362:1022-9.

80. Nickerson EK, Sinha R. Vertebral osteomyelitis in adults: an update. Br Med Bull 2016;117:121-38.

81. Berbari EF, Kanj SS, Kowalski TJ, et al. 2015 Infectious Diseases Society of America (IDSA) clinical practice guidelines for the diagnosis and treatment of native vertebral osteomyelitis in adults. Clin Infect Dis 2015;61:e26-46.

82. Chenoweth CE, Bassin BS, Mack MR, et al. Vertebral osteomyelitis, discitis, and spinal epidural abscess in adults. Ann Arbor (MI): Michigan Medicine University of Michigan; 2018.

83. Bingham JS, Hassebrock JD, Christensen AL, Beauchamp CP, Clarke HD, Spangehl MJ. Screening for periprosthetic joint infections with ESR and CRP: the ideal cutoffs. J Arthroplasty 2020;35:1351-4.

84. Pannu TS, Villa JM, Patel PD, Riesgo AM, Barsoum WK, Higuera CA. The utility of serum d-dimer for the diagnosis of periprosthetic joint infection in revision total hip and knee arthroplasty. J Arthroplasty 2020;35:1692-5.

85. Povoa P. C-reactive protein: a valuable marker of sepsis. Intensive Care Med 2002;28:235-43.

86. Jeong DK, Lee HW, Kwon YM. Clinical value of procalcitonin in patients with spinal infection. J Korean Neurosurg Soc 2015;58:271-5. 
87. Li HK, Rombach I, Zambellas R, et al. Oral versus intravenous antibiotics for bone and joint infection. N Engl J Med 2019;380:425-36.

88. Baek KH, Lee YS, Kang DH, Lee CH, Hwang SH, Park IS. The safety and decision making of instrumented surgery in infectious spondylitis. Korean J Spine 2016;13:120-3.

89. Park KH, Cho OH, Lee YM, et al. Therapeutic outcomes of hematogenous vertebral osteomyelitis with instrumented surgery. Clin Infect Dis 2015;60:1330-8.

90. Talia AJ, Wong ML, Lau HC, Kaye AH. Safety of instrumentation and fusion at the time of surgical debridement for spinal infection. J Clin Neurosci 2015;22:1111-6.

91. Dai LY, Chen WH, Jiang LS. Anterior instrumentation for the treatment of pyogenic vertebral osteomyelitis of thoracic and lumbar spine. Eur Spine J 2008;17:1027-34.

92. Chen WH, Jiang LS, Dai LY. Surgical treatment of pyogenic vertebral osteomyelitis with spinal instrumentation. Eur Spine J 2007;16:1307-16.

93. Hsieh PC, Wienecke RJ, O’Shaughnessy BA, Koski TR, Ondra SL. Surgical strategies for vertebral osteomyelitis and epidural abscess. Neurosurg Focus 2004;17:E4.

94. Dennis Hey HW, Nathaniel Ng LW, Tan CS, et al. Spinal implants can be inserted in patients with deep spine infection: results from a large cohort study. Spine (Phila Pa 1976) 2017;42:E490-5.

95. Pee YH, Park JD, Choi YG, Lee SH. Anterior debridement and fusion followed by posterior pedicle screw fixation in pyogenic spondylodiscitis: autologous iliac bone strut versus cage. J Neurosurg Spine 2008;8:405-12.

96. Carragee E, Iezza A. Does acute placement of instrumentation in the treatment of vertebral osteomyelitis predispose to recurrent infection: long-term followup in immune-suppressed patients. Spine (Phila Pa 1976) 2008;33:2089-93.

97. Ghobrial GM, Viereck MJ, Margiotta PJ, et al. Surgical management in 40 consecutive patients with cervical spinal epidural abscesses: shifting toward circumferential treatment. Spine (Phila Pa 1976) 2015;40:E949-53.

98. Ghobrial GM, Beygi S, Viereck MJ, et al. Timing in the surgical evacuation of spinal epidural abscesses. Neurosurg Focus 2014;37:E1.
99. Epstein NE. Timing and prognosis of surgery for spinal epidural abscess: a review. Surg Neurol Int 2015;6(Suppl 19):S475-86.

100. DePasse JM, Ruttiman R, Eltorai AE, Palumbo MA, Daniels AH. Assessment of malpractice claims due to spinal epidural abscess. J Neurosurg Spine 2017;27:476-80.

101. French KL, Daniels EW, Ahn UM, Ahn NU. Medicolegal cases for spinal epidural hematoma and spinal epidural abscess. Orthopedics 2013;36:48-53.

102. Shantharam G, DePasse JM, Eltorai AE, Durand WM, Palumbo MA, Daniels AH. Physician and patients factors associated with outcome of spinal epidural abscess related malpractice litigation. Orthop Rev (Pavia) 2018;10:7693.

103. Ju KL, Kim SD, Melikian R, Bono CM, Harris MB. Predicting patients with concurrent noncontiguous spinal epidural abscess lesions. Spine J 2015;15:95101.

104. Avanali R, Ranjan M, Ramachandran S, Devi BI, Narayanan V. Primary pyogenic spinal epidural abscess: how late is too late and how bad is too bad?: a study on surgical outcome after delayed presentation. Br J Neurosurg 2016;30:91-6.

105. Grewal S, Hocking G, Wildsmith JA. Epidural abscesses. Br J Anaesth 2006;96:292-302.

106. Duarte RM, Vaccaro AR. Spinal infection: state of the art and management algorithm. Eur Spine J 2013;22:2787-99.

107. Urrutia J, Zamora T, Campos M. Cervical pyogenic spinal infections: are they more severe diseases than infections in other vertebral locations? Eur Spine J 2013;22:2815-20.

108. O'Brien C, Lenehan B, Street J. Non-operative management of an extensive anteriorly located epidural abscess. J Clin Neurosci 2011;18:1401-2.

109. Uchida K, Nakajima H, Yayama T, et al. Epidural abscess associated with pyogenic spondylodiscitis of the lumbar spine; evaluation of a new MRI staging classification and imaging findings as indicators of surgical management: a retrospective study of 37 patients. Arch Orthop Trauma Surg 2010;130:111-8.

110. Carr C, Diehn F, Kaufmann T. Nonenhancing epidural abscess and discitis in an immunocompromised patient: a case report. Clin Neurol Neurosurg 2013;115:2274-6. 